\title{
Comparing ice composition in dark molecular clouds
}

\author{
C. Knez ${ }^{1,2}$, M. Moore ${ }^{2}$, S. Travis ${ }^{3}$, R. Ferrante ${ }^{3}$, J. Chiar ${ }^{4}$, \\ A. Boogert ${ }^{5}$, L. Mundy ${ }^{1,2}$, Y. Pendleton ${ }^{6}$, A. Tielens ${ }^{7}$, \\ E. van Dishoeck ${ }^{8}$, and N. Evans ${ }^{9}$ \\ ${ }^{1}$ University of Maryland, email: claudia@astro.umd.edu, ${ }^{2}$ Goddard Center for Astrobiology, \\ ${ }^{3}$ U.S. Naval Academy, ${ }^{4}$ SETI Institute, ${ }^{5}$ Herschel Science Center, ${ }^{6}$ NASA Headquarters, \\ ${ }^{7}$ NASA Ames, ${ }^{8}$ Leiden University, ${ }^{9}$ University of Texas at Austin
}

\begin{abstract}
We present 5-20 $\mu \mathrm{m}$ Spitzer/IRS spectroscopy toward stars behind dark molecular clouds. We present preliminary results from the Serpens dark cloud to show the variation between environments within a cloud. We are surveying 3 clouds with varying levels of star formation activity. Serpens has the highest level of activity from our 3 clouds. We show that location as well extinction can cause variations in ice composition. We also find that some lines of sight contain organic molecules such as methane and methanol, and the first detection of acetylene ice in the interstellar medium. We believe the high extinction lines of sight have been enriched by star formation activity near those lines of sight.
\end{abstract}

Keywords. ISM: molecules, astrochemistry, techniques: spectroscopic

\section{Introduction}

Infrared spectroscopy of protostars and extincted background stars has revealed the presence of numerous features due to simple molecules frozen on refractory dust grains: $\mathrm{H}_{2} \mathrm{O}, \mathrm{CO}_{2}, \mathrm{CO}, \mathrm{CH}_{3} \mathrm{OH}, \mathrm{CH}_{4}, \mathrm{HCOOH}, \mathrm{OCN}^{-}$, and possibly $\mathrm{NH}_{3}$ and $\mathrm{NH}_{4}^{+}$(e.g., Whittet et al. 1996, Schutte et al. 1996). Several key species $\left(\mathrm{H}_{2} \mathrm{O}, \mathrm{CH}_{3} \mathrm{OH}\right)$ are formed by chemical reactions on the grains, while others $(\mathrm{CO})$ accrete inertly from the gas phase. Evidently, the composition of these icy grain mantles is directly related to the composition of the gas (e.g., the $\mathrm{C} / \mathrm{CO}$ ratio), which in turn is related to cloud history. Laboratory irradiation studies of simple ices have shown that quite complex species can be formed this way (PAHs, amino acids, hydrocarbons; Bernstein et al. 2002, Greenberg et al. 2000). Eventually, the ices may be released back in the gas phase, driving a chemistry capable of forming complex species in the 'hot cor(ino)es' surrounding protostars (Cazaux et al. 2003). Alternatively, they may be incorporated in icy bodies and deliver volatiles to planetesimals in circumstellar disks. Knowledge of the ice composition and the relevance of the various molecule formation and destruction processes is thus key to understanding interstellar and protoplanetary chemistry.

The composition of interstellar ices is expected to reflect the local conditions in the dense regions of the clouds. Specifically, gas density and dust temperature are thought to be important drivers of grain surface chemistry (Tielens \& Hagen 1982, Hasegawa \& Herbst 1993, Bergin et al. 1997). These physical conditions drive the star formation activity in the cloud core and in turn newly formed stars affect the density and dust temperature. Hence, the composition of the accreted ice mantles is expected to vary with the star formation activity of the cloud.

As part of a Spitzer GO-4 program, we are obtaining 5-20 $\mu \mathrm{m}$ spectra toward stars behind the Serpens, Perseus, and Lupus dark clouds. Spitzer has greatly enhaced the 
Table 1. Abundances towards two lines of sight with high $A_{V}$

\begin{tabular}{l|cc|c|c}
\hline Species & Unit & CK 2 & Ser BG1 \\
\hline $\mathrm{CO}_{2}$ & $\% \mathrm{H}_{2} \mathrm{O}$ & 33 & 35 \\
$\mathrm{CH}_{3} \mathrm{OH}$ & $\% \mathrm{H}_{2} \mathrm{O}$ & $<2.1$ & 17 \\
$\mathrm{CH}_{4}$ & $\% \mathrm{H}_{2} \mathrm{O}$ & $<3$ & $\sim 3$ \\
$\mathrm{C}_{2} \mathrm{H}_{2}$ & $\% \mathrm{H}_{2} \mathrm{O}$ & $\ldots$ & $\sim 3$ \\
$\mathrm{HCOOH}$ & $\% \mathrm{H}_{2} \mathrm{O}$ & 1.9 & 2.6 \\
\hline
\end{tabular}

study of ice composition toward molecular clouds by being able to probe lines of sight toward faint young stellar objects as well as toward faint stars behind the clouds. We conducted follow up observations of the brightest extincted stars behind three of the clouds mapped by the c2d legacy team: Serpens (high star formation activity), Perseus (medium activity), and Lupus (low activity).

\section{Observations and results}

In this paper, we analyze a sample of 10 stars in Serpens to study the ice composition to determine what factors play a role in the icy grain mantle composition. The preliminary sample shows that the $6 \mu \mathrm{m}$ feature increases in depth with extinction but it is not necessarily larger at the highest extinction. The depth of the $6 \mu \mathrm{m}$ feature generally increases with $A_{V}$ but there are variations especially at low and at high extinctions. Some variations in the $6 \mu \mathrm{m}$ feature may due to the contribution of other species such as $\mathrm{HCOOH}$, especially at high extinction.

We compared two highly extincted lines of sight in Serpens to see if both sight-lines showed similar composition. CK 2 has been analyzed by Knez et al. (2005). It has an $A_{V}$ greater than $30 \mathrm{mag}$ and shows many ice features with only upper limits on organics such as $\mathrm{CH}_{4}$ and $\mathrm{CH}_{3} \mathrm{OH}$ (see Table 1). The other sight-line is toward Ser BG1 with an $A_{V} \sim 25$ mag. This sources has a lower extinction than CK 2 yet shows higher column densities of the following organics: $\mathrm{CH}_{4}$ and $\mathrm{CH}_{3} \mathrm{OH}$. In addition, this is the first source in which solid $\mathrm{C}_{2} \mathrm{H}_{2}$ has been detected in space.

\section{Conclusions}

We present the first detection of $\mathrm{C}_{2} \mathrm{H}_{2}$ ice in the interstellar medium. It has been detected with comparable abundance to $\mathrm{CH}_{4}$. Extinction is not the only variable in determining the composition of ices in molecular clouds. Even toward lines of sight where there are no protostars, the effects of star formation can affect the grain composition.

\section{References}

Bergin, E. A., et al. 1997, ApJ, 482, 285

Bernstein, M. P., et al. 2002, ApJ, 576, 1115

Cazaux, S., et al. 2003, ApJL, 593, 51

Greenberg, J. M., et al. 2000, ApJL, 531, 71

Hasegawa, T. I. \& Herbst, E. 1993, MNRAS, 263, 589

Knez, C., et al., 2005, ApJL, 635, 145

Schutte, W., et al. 1996, A\&A, 309, 633

Tielens, A. G. G. M. \& Hagen, W. 1982, $A \mathscr{E} A$, 114, 245

Whittet, D. C. B., et al. 1996, ApJ, 458, 363 